\title{
Mettre en mots, en nombres et en politique la nature néo-calédonienne
}

\section{Elsa Faugère}

\section{(2) OpenEdition \\ Journals}

Édition électronique

URL : http://journals.openedition.org/jso/3742

DOI : $10.4000 /$ jso.3742

ISSN : $1760-7256$

Éditeur

Société des océanistes

\section{Édition imprimée}

Date de publication : 15 décembre 2008

Pagination : 153-166

ISBN : 978-2-85430-012-3

ISSN : 0300-953x

\section{Référence électronique}

Elsa Faugère, « Mettre en mots, en nombres et en politique la nature néo-calédonienne », Journal de la Société des Océanistes [En ligne], 126-127 | Année 2008, mis en ligne le 15 décembre 2011, consulté le 17 juin 2020. URL : http://journals.openedition.org/jso/3742 ; DOl : https://doi.org/10.4000/jso.3742 


\section{Mettre en mots, en nombres et en politique la nature néo-calédonienne}

par

Elsa FAUGÈRE*

\section{RÉSUMÉ}

L'anthropologie s'est toujours intéressée aux rapports entre les sociétés humaines et leurs ressources naturelles. Plus récemment, l'anthropologie a exploré la notion de nature-patrimoine, tendant ainsi à constituer une anthropologie de l'environnement encore en formation. Cet article se situe dans ce cadre-là. En prenant comme point de départ et d'interrogation l'existence du Programme de conservation des forêts sèches de Nouvelle-Calédonie, cet article adopte une perspective historique pour tenter de comprendre comment et pourquoi la nature néo-calédonienne figure, depuis la fin des années 1980 et le milieu des années 1990, dans la liste de deux principaux classements internationaux en matière de conservation de la biodiversité : celui de Conservation International avec la notion de hotspot et celui du WWF avec la notion d'éco-régions. Se basant sur une analyse de textes et quelques entretiens, cet article tente de poser des jalons de l'histoire de la botanique en Nouvelle-Calédonie centrée sur les opérations de mise en mots et en nombres de la nature. L'intense et ancienne exploration botanique de la Nouvelle-Calédonie permet de mieux comprendre pourquoi, dans les années 19801990, les forêts sèches ont fait l'objet d'une attention scientifique particulière et conduit à la mise en alerte des pouvoirs publics locaux ainsi que d'une oNG internationale de la conservation, le wwF.

Mots-CLÉs : Nouvelle-Calédonie, forêts sèches, conservation, biodiversité, histoire, botanique

\begin{abstract}
Interactions between human societies and their natural environment have always been studied by anthropologists. But their focus was more on natural resources than on nature as patrimony. More recently, some anthropologists have begun to study nature as a patrimony, leading to a new anthropology of environment. This paper adopts such a perspective. The starting point is the conservation program of dry forests of New Caledonia. The question is: why biodiversity of New Caledonia was listed in two main international conservation programs at the end of the eighties and the mid of the nineties? Why Conservation International has called it a hotspot of biodiversity, and the WWF an Eco-Region? By analyzing several documents and interviews, this paper tries to outline a brief history of botanical science in New Caledonia which is quite ancient and intense. It helps us to better understand why, in the eighties and nineties, dry forests were particularly well studied by the natural scientists whose works have alerted both local authorities and an international ${ }_{N G O}$, the WWF.
\end{abstract}

KEYwORDS: New Caledonia, dry forests, conservation, biodiversity, history, botany 


\section{Anthropologie économique et nature}

Entre autres significations, le mot économie désigne « l'ensemble des faits relatifs à la production, à la distribution et à la consommation des richesses dans une collectivité » (Le Petit Robert, 2004 : 828). Parmi les richesses d'une collectivité, les ressources naturelles occupent une place majeure. Sans elles, il n'existerait ni économie, ni société, ni vie humaine. Les anthropologues qui étudient la vie économique des groupes sociaux ne peuvent donc se désintéresser de la place et du rôle de la nature dans le fonctionnement des sociétés qu'ils étudient. Pourtant, de nombreux auteurs ont placé au cœur de leurs enquêtes ethnographiques et de leurs questionnements anthropologiques la circulation des biens ou des richesses, naturelles et non naturelles, sans donner à la nature la place centrale qu'elle occupe dans les phénomènes étudiés.

$\mathrm{Au}$ cours des enquêtes ethnographiques que j'ai menées sur l'île de Maré1, entre le mois de juillet 1994 et le mois de mai 1997, je me suis particulièrement intéressée aux transactions monétaires et non monétaires, marchandes et non marchandes, qui s'effectuent lors des grandes cérémonies dites coutumières: mariages, deuils et demandes en mariages. Lors de ces événements, importants dans la vie sociale et économique locale, de nombreux biens circulent au sein des réseaux de parenté : billets de banque, ignames, poissons, morceaux de bétail, paquets de riz, poulets congelés, huile, etc. Ces biens vont de produits bruts et "sauvages» (très peu transformés par l'action de l'homme) à des produits moins bruts et plus domestiqués (les plus transformés par l'action de l'homme). On aurait ainsi, à une extrémité, les poissons sauvages pêchés dans le lagon et en mer, produits d'une prédation de l'homme sur la nature sans domestication et, à l'autre extrémité, les billets de banque, fabriqués à partir d'une ressource naturelle (des arbres) transformés tout au long d'une chaîne complexe associant une pluralité d'acteurs, collectifs et individuels, situés dans des lieux et des institutions éloignés les uns des autres. Entre les deux, on trouverait les ignames et le bétail, objets d'une nature domestiquée et cultivée sur place, puis les paquets de riz, les poulets congelés et l'huile, produits d'une nature cultivée ailleurs et transformée dans des usines agroalimentaires
L'origine naturelle, totale ou partielle, de ces différents objets, leurs différents degrés de « naturalité », pourrait-on dire, ne m'étaient pas aussi sensibles et présents à l'époque de mes enquêtes ethnographiques qu'aujourd'hui. Je m'intéressais alors à d'autres aspects de ces phénomènes. Aujourd'hui, je m'étonne de l'invisibilité de la nature et de la matérialité naturelle de ces objets qui étaient pourtant au cœur de mes investigations. Des sociologues comme Michel Callon, John Law et Bruno Latour ont justement attiré l'attention sur l'absence d'objets qu'ils appellent les «non-humains » dans les travaux en sciences sociales (voir notamment Latour, 1997).

Si les analyses de ces auteurs sont convaincantes et séduisantes, il convient cependant de nuancer leur constat pour l'anthropologie qui s'est très tôt et longtemps intéressée à ce qu'on appelle " la culture matérielle». Les anthropologues ne se sont pas désintéressés des "non-humains» qui ont constitué pendant des décennies un des axes majeurs de leurs recherches. En outre, la question des frontières entre nature et cultures, c'est-à-dire entre "non-humains » et humains, est constitutive de l'anthropologie. Pourtant, force est de constater qu'il est possible de s'intéresser aux objets d'une nature plus ou moins domestiquée ou plus ou moins sauvage, sans pour autant être nécessairement conduit à reconnaître l'origine naturelle des objets ou des processus, c'est-à-dire à prendre en compte la présence de la nature dans ces objets et processus. Une telle lecture peut être comparée, en miroir, à celle des écologues lorsqu'ils ne voient pas, dans les processus et phénomènes naturels qu'ils étudient, les marques plus ou moins visibles des activités humaines.

Que les ethnologues ne prennent pas en compte la nature dans les phénomènes sociaux qu'ils examinent et que les écologues ne voient pas la culture dans les phénomènes naturels qu'ils étudient renvoie à l'histoire des représentations occidentales de la nature et de la culture et à leur traduction dans l'histoire des sciences. L'opposition faite en Occident entre nature et culture se retrouve en effet dans le découpage sciences de la nature versus sciences de la culture et de la société - des objets d'étude, "naturels » ou « culturels $»^{2}$, de ces disciplines.

Si certains auteurs ont pratiqué l'anthropologie (économique) sans se soucier de la nature, d'autres l'ont bien prise en compte, comme en

1. L'île de Maré fait partie de la province des îles Loyauté.

2. De très nombreux travaux, d'historiens notamment, ont souligné et analysé l'opposition faite en Occident entre nature et culture. 
témoignent certains travaux de l'anthropologie économique française des années 1960-1970. Sans entrer dans les détails de ce foisonnant courant anthropologique, la lecture des travaux montre bien que la nature est omniprésente tout en étant centrée sur une modalité particulière du rapport homme-nature : celle de l'exploitation. Les concepts de modes et de rapports de production renvoient à une nature considérée comme une nature à exploiter, à domestiquer, à faire fructifier pour les besoins et les profits économiques et sociaux des humains. Cette nature est une nature-ressource. Une telle anthropologie, que l'on pourrait appeler des ressources naturelles, ne date pas des années 1960. On la trouve déjà en 1921 chez Bronislaw Malinowski dans L'économie primitive des îles Trobriand où l'auteur écrit, au début de son article :

« Je commencerai par brosser un tableau général des ressources naturelles des Trobriandais et par décrire dans ses grandes lignes la manière dont celles-ci sont exploitées. » (in Godelier, 1974 : 81)

C'est cette nature-ressource que les économistes appellent une « nature-capital ». Ils opposent ou distinguent cette conception de la nature d'une autre conception centrée sur la transmission de la nature aux générations futures, c'està-dire sur une conception de la nature en tant que patrimoine à protéger et à transmettre. Il s'agit là d'une "nature-patrimoine» (Vivien, 2001). On peut ainsi distinguer une anthropologie des ressources naturelles présente dès les débuts de l'anthropologie, qui est plus particulièrement au cœur des préoccupations d'une certaine anthropologie économique moderne, et une anthropologie se préoccupant plus attentivement de la « nature-patrimoine».

L'anthropologie de l'environnement regroupe ces deux approches et met en évidence, en particulier, leur indissociabilité. C'est une anthropologie du « souci de l'environnement », à ses trois échelles de temps principales, le présent (la ressource) et le passé et l'avenir (le patrimoine). Le développement de cette approche anthropologique de l'environnement a suivi l'essor des préoccupations écologiques et environnementales dans les sociétés occidentales au cours des années 1960-1970. Cet article reflète ces développements parallèles. La distinction que je viens de faire entre ces deux traitements anthropologiques de la nature ne signifie pas qu'il n'en existe pas d'autres ni que l'on ne puisse pas se situer entre les deux. Concilier et articuler les deux modalités du rapport entre nature et culture demeurent toujours l'un des enjeux de l'anthropologie contemporaine.

\section{Conserver la biodiversité ou « rééquilibrer » la Nouvelle-Calédonie ?}

C'est précisément en m'intéressant aux questions environnementales en Nouvelle-Calédonie que j'ai eu la surprise de découvrir, sur Internet, un texte de trente pages du wwF (Fonds mondial pour la nature), daté de janvier 2000 et intitulé : Programme de conservation. Forêts tropicales sèches en Nouvelle-Calédonie. Outre le fait que j'apprenais ainsi l'importance internationalement reconnue de la biodiversité néo-calédonienne, point qui m'avait totalement échappé au cours de mes enquêtes ethnographiques, je lisais avec stupeur que certaines des terres sur lesquelles portait ce programme de conservation, d'envergure internationale, appartenaient à de grands propriétaires terriens d'origine européenne.

Je n'étais pas revenue en Nouvelle-Calédonie depuis 1998, date de la signature de l'accord de Nouméa qui avait repris à son compte l'objectif de "rééquilibrage» mis à l'honneur par les accords de Matignon signés dix années auparavant. Le simple fait qu'une action publique, en l'occurrence un important programme de conservation de la nature, puisse se situer sur les terres de grands propriétaires terriens d'origine européenne et donc leur bénéficier, me paraissait remettre en question ou tout au moins interroger la notion et l'objectif même du rééquilibrage. En effet, le principe de base du rééquilibrage était de rétablir une certaine justice sociale en faveur des populations kanak spoliées et dépossédées par la colonisation française. Cette dernière avait produit des différences de développement entre la province Sud, blanche, urbaine et développée et les provinces Nord et Îles Loyauté, majoritairement peuplées de Kanak et considérées comme des zones rurales sous-développées. Pour rétablir un équilibre, il fallait donc mettre en œuvre des politiques de développement bénéficiant prioritairement aux Kanak de ces zones rurales sousdéveloppées.

Mais ce programme de conservation des forêts sèches semblait proposer un tout autre type d'actions dirigées vers la préservation de la biodiversité et situées sur des terres européennes. Cela signifiait-il, qu'au nom de la conservation de la nature, en l'occurrence de forêts sèches, on pouvait mettre de côté, voire transgresser, le « juste» objectif du rééquilibrage? Ou, plus encore, cela signifiait-il que la conservation de la nature pouvait servir de prétexte, de caution ou d'alibi pour conserver ainsi des rapports sociaux de domination? La conservation de la nature était-elle placée au-dessus de la justice sociale ou 
servait-elle plutôt à conserver des injustices sociales?

Ces interrogations ont constitué le point de départ de mon enquête. Celle-ci s'intègre dans un programme de recherche sur la conservation de la biodiversité appréhendée sous deux angles différents : celui de la constitution de connaissances, savoirs scientifiques naturalistes quantitatifs (reposant sur la mesure des quantités et des flux de matières végétales), méthodes modernes de mesure de la biodiversité, et celui des réseaux et des modes de financement internationaux (comment finance-t-on la conservation de la biodiversité ?). Les questions de conservation de la biodiversité, néo-calédonienne en l'occurrence, conduisent à s'intéresser aux savoirs scientifiques naturalistes qui sont les bases indispensables à l'élaboration des politiques de conservation. L'histoire des sciences naturelles en Nouvelle-Calédonie montre l'ancienneté de la constitution d'un savoir scientifique sur la nature néo-calédonienne qui a permis de classer, dans les années 1990, la biodiversité néo-calédonienne parmi les plus remarquables et les plus menacées du monde. Comment se sont, historiquement, construits un intérêt et un savoir scientifiques et politiques sur la nature en NouvelleCalédonie ? Comment comprendre que dans les années 1990, la biodiversité néo-calédonienne ait pu figurer dans la liste de plusieurs classements internationaux en matière de conservation de la nature, notamment :

- Le document Global 200 du wwF (Olson et al., 2000) où 238 éco-régions sont identifiées dans le but d'en préserver la biodiversité et les écosystèmes, considérés comme particulièrement précieux. Quatre éco-régions de la NouvelleCalédonie figurent dans ce document : l'écorégion 18 « forêts humides »; l'éco-région 53 " forêts sèches "; l'éco-région 166 « rivières et ruisseaux »; l'éco-région 221 "barrières de corail ». Ces 238 éco-régions constituent les exemples les plus remarquables et les plus représentatifs de la diversité des écosystèmes mondiaux. Ce sont des régions prioritaires pour des actions de conservation.

- La liste des hotspots, foyers de la biodiversité mondiale affichée dans le classement de l'oNG Conservation International contient aussi des sites néo-calédoniens. En 1988, dans le but d'identifier des sites prioritaires pour les politiques de conservation de la nature, l'écologue britannique Norman Myers (1988) définit le concept de hotspot de la biodiversité. Il retient deux facteurs pour qualifier un territoire de hotspot : la quantité d'espèces végétales endémiques $^{3}$ présentes sur ce territoire et l'impact des activités humaines sur sa végétation primaire. Pour être qualifiée de hotspot de la biodiversité, une région doit contenir au moins mille cinq cents espèces de plantes vasculaires ${ }^{4}$ endémiques et avoir perdu au moins $70 \%$ de sa couverture végétale primaire (Myers, 1988). L'élaboration du concept de hotspot de la biodiversité s'appuie donc sur un registre argumentaire qui mêle des opérations de comptage d'espèces naturelles, des traitements statistiques et des discours alarmistes. Ce sont précisément ces opérations de quantification et de mesure (comptages d'espèces et traitements statistiques) qui m’intéressent ici.

Le défi auquel conduisait l'article de Norman Myers était alors le suivant : comment savoir sur quels territoires, sur quels espaces et sur quelles espèces les politiques de conservation de la nature devaient se tourner prioritairement? Le concept de hotspot de la biodiversité devait précisément permettre de hiérarchiser les priorités conservationnistes. Dans cet article, Norman Myers se basait sur les forêts tropicales primaires où il distinguait dix points chauds ou hauts lieux de la biodiversité5. Les forêts sèches et humides de Nouvelle-Calédonie figuraient parmi ces dix hotspots de la biodiversité mondiale.

\section{Méthodologie}

Pour comprendre par quels cheminements la nature néo-calédonienne, portée à la connaissance des Européens à partir du mois de septembre 1774, figure, depuis la fin des années 1980, dans la liste des sites internationaux de conservation de la biodiversité, il est nécessaire de retracer brièvement l'histoire des sciences naturelles en Nouvelle-Calédonie. Je ne ferai que poser ici les premiers jalons de cette histoire. Ce faisant, je tenterai de mettre en évidence quelques-unes des démarches - descriptions systématiques et traitements quantitatifs des don-

3. Le terme « endémique qualifie le fait qu'une espèce vivante est exclusivement inféodée à une aire biogéographique donnée, en général de faible étendue » (Ramade, $1993: 224$ ).

4. "Les plantes vasculaires sont les végétaux supérieurs possédant des vaisseaux irriguant les tiges, les racines et les feuilles » (Le Petit Robert, 2004 : 2741).

5. En 1988, les dix points chauds de la biodiversité identifiés par Norman Myers étaient les suivants : Madagascar ; Brazil Atlantic Forest; Western Ecuador; Colombian Choco; Western Amazonian Uplands ; Eastern Himalayas ; Peninsular Malaysia ; Northern Borneo ; Philippines ; New Caledonia. 
nées - que les scientifiques mettent en œuvre pour élaborer le savoir naturaliste et quels registres argumentaires ils utilisent pour alerter les pouvoirs publics et les bailleurs de fonds. Je me suis focalisée sur l'étude de la végétation puisqu'elle a toujours été l'un des objets de l'intérêt des naturalistes européens en NouvelleCalédonie depuis le XVIII ${ }^{\mathrm{e}}$ siècle et qu'elle permet d'illustrer le concept de hotspot de la biodiversité élaboré par Norman Myers en 1988.

«Dans son premier article sur la biodiversité des hotspots, Myers a utilisé les végétaux comme indicateurs de cette biodiversité. » (Mittermeier et al., 1998 : 516 , traduit de l'anglais par moi)

Pour commencer à retracer cette histoire, j'ai constitué un corpus de différentes sortes de textes trouvés pour la plupart sur Internet :

- des archives de revues scientifiques coloniales mises en ligne sur le site de Gallica, bibliothèque numérique de la Bibliothèque nationale de France (http://gallica.bnf.fr) ;

- le fonds documentaire de l'IRD qui offre plusieurs milliers de publications en ligne des chercheurs de l' IRD (http://www.bondy.ird. fr/pleins_textes/index-tous.htm).

Je me suis également basée sur plusieurs entretiens que j'ai eus en 2005 avec des botanistes et des naturalistes de l'IRD et du Muséum national d'histoire naturelle de Paris (Philippe Bouchet, Philippe Morat, Tanguy Jaffré, Jean-Marie Veillon et Bernard Suprin), ainsi qu'avec le directeur du Programme de conservation des forêts sèches de Nouvelle-Calédonie (Christian Papineau).

\section{Mettre la nature en mots savants}

Historiquement et logiquement, les opérations de reconnaissance et de classification des espèces végétales ont précédé les opérations de quantification, de mesure et de traitement statistique de la nature néo-calédonienne. Si pour évaluer le nombre d'espèces végétales présentes sur un territoire, il faut pouvoir les compter, une telle opération de comptage présuppose l'existence d'un savoir naturaliste préalable incluant la reconnaissance et le classement des espèces.

\section{Les premières explorations botaniques: 1774- 1853}

C'est en septembre 1774 que ce travail commence en Nouvelle-Calédonie, lors du deuxième voyage de circumnavigation de James Cook. À son bord, plusieurs savants, dont un astronome, William Wales, et trois naturalistes, Johann Reinhold Forster, son fils George Forster et Anders Spaarman, médecin suédois, élève et disciple du célèbre naturaliste Linné. Leur exploration terrestre de cette terre, qu'ils appelleront New Caledonia, fut très breve : sept jours et demi, selon Georges Forster (Pisier, 1983 : 105). Il marque le début de la construction d'un savoir scientifique sur les objets naturels de l'île (flore, faune, minéraux...). Comme le raconte laconiquement James Cook (Pisier, 1983 : 39), ces naturalistes partaient herboriser. Ils collectaient des échantillons de végétaux dans le but de les décrire, à la fois par des mots et par des dessins. Ces mises en mots et en formes de la nature constituent une étape essentielle dans la construction d'un savoir naturaliste scientifique. La création d'un lexique descriptif commun suivie d'une phase taxonomique, c'est-à-dire de classification de ces objets naturels ainsi décrits, caractérise l'histoire naturelle du XVIII ${ }^{\mathrm{e}}$ siècle. Pour connaître et ensuite reconnaître la diversité du vivant découverte au cours de ces grands voyages, un travail laborieux et complexe de désignation et de classification a été effectué auX $\mathrm{XVIII}^{\mathrm{e}}$ et $\mathrm{XIX}^{\mathrm{e}}$ siècles. Ces étapes fondatrices du savoir naturaliste scientifique ont été largement décrites et étudiées par les historiens des sciences (Drouin, 1997: 479-501; Blanckært et al., 1997 : 163-196).

" Amorcée dès le XVII ${ }^{\mathrm{e}}$ siècle, l'aventure des voyageurs naturalistes connaît son apogée à la fin du XVIII ${ }^{\mathrm{e}}$ siècle et au début du XIX ${ }^{\mathrm{e}}$. » (Drouin, $1997: 480$ )

«Les naturalistes, de retour de leurs périples n'apportaient pas que des récits, ils revenaient chargés d'espèces jusqu'alors inconnues : dépouilles d'animaux exotiques, et surtout feuilles d'herbiers accompagnés de graines et parfois de plants en pots. » (Drouin, 1997 : 486)

Selon Jean-Marc Drouin (1997 : 486), si l'on s'en tient uniquement au règne végétal, le nombre d'espèces connues au XVI ${ }^{\mathrm{e}}$ siècle et décrites par les botanistes ne dépasse pas quelques milliers, alors qu'en 1833, on en connaît plus de cinquante mille et plus de deux cent cinquante mille en 1997. Mais, poursuit Jean-Marc Drouin (ibid. : 487-88) :

« de tels dénombrements ne vont pas de soi. Ils supposent des spécimens récoltés, préparés, dessinés, décrits, rassemblés en des lieux, musées, jardins, herbiers, cabinets d'histoire naturelle où chacun puisse venir les voir, les observer, les comparer... Voyages et collections apparaissent ainsi comme les deux pôles de l'histoire naturelle. Toutefois, entre ces deux pôles, rien ne passerait si on ne se mettait pas en peine de nommer et classer tous les spécimens apportés. Entre l'aventure 
des voyages et la poésie des jardins, la nomenclature et la classification [...] forment l'échangeur qui en les reliant l'un à l'autre, conditionne la production d'un savoir sur le vivant. » (1997 : 487-488)

Ce sont donc les trois naturalistes présents à bord du navire de James Cook qui les premiers firent observations, prélèvements, descriptions et qui rapportèrent en Europe les premiers échantillons de la flore néo-calédonienne. On en trouve aujourd'hui trace, non seulement dans leurs récits de voyage, mais aussi dans leurs publications scientifiques et dans des articles contemporains qui reprennent la nomenclature utilisée par ces premiers naturalistes. Ces premiers écrits et, notamment, le récit de voyage de George Forster, insistent déjà sur le caractère remarquable de la fore néo-calédonienne et sur l'intérêt qu'il y aurait à l'étudier pour l'Histoire naturelle. James Cook note d'ailleurs dans son journal :

« Nos botanistes n'eurent pas à se plaindre de manquer d'occupation ici ; chaque jour apportait quelque chose de nouveau en botanique ou dans les autres branches de l'histoire naturelle. » (Pisier, 1983 : 48)

George Forster souligne à plusieurs reprises dans son récit de voyage l'intérêt pour la science de la découverte qu'ils firent de nouvelles espèces végétales et animales. Il conclut son récit en disant que :

" les animaux, les végétaux et les minéraux [de la Nouvelle-Calédonie] qui demeurent inviolés, offrent un ample champ d'étude à un naturaliste. » (Pisier, $1983: 117)$

La nature néo-calédonienne apparaît donc, dès les toutes premières prospections et collections, comme un riche objet d'étude scientifique. Les récits d'Anders Spaarman écrits plus tardivement, en 1802 et en 1818, le confirment. Dans les larges extraits publiés par Georges Pisier, il en souligne également la richesse botanique et, notamment, celle d'une île qu'ils appelèrent «l'île Botanique " en raison de la découverte qu'ils y firent d'une trentaine de nouvelles espèces. Ces toutes premières descriptions naturalistes et plus précisément celles des Forster soulevèrent une controverse scientifique entre l'astronome William Wales, qui était également à bord de la Resolution, et les Forster. William Wales contesta en effet l'exactitude des observations effectuées par George Forster (Pisier, 1983 : 121). Cette controverse, qui reste à étudier en détails, est racontée dans un article de M. E. Hoare paru en 1967 (cité par Pisier, 1983). Tous ces écrits ont contribué à enrichir la connaissance de la nature au XVIII ${ }^{\mathrm{e}}$ siècle et ont fourni de précieux éléments sur la nature néo-calédonienne, sur sa richesse végétale et sur la présence d'espèces inconnues jusqu'alors. Mais c'est La Billardière, botaniste français et naturaliste de l'expédition d'Entrecasteaux (1793), qui publia en 1800 le premier ouvrage important et significatif sur la botanique de la Nouvelle-Calédonie.

Ces premiers textes scientifiques sur la nature néo-calédonienne restèrent isolés pendant plus d'un demi-siècle. La France relança, sous la Restauration, les voyages de circumnavigation dans le Pacifique (Merle, 1995: 35) et organisa six grandes expéditions entre 1816 et 1832 (Merle, 1995 : 409). Mais aucune d'entre elles ne s'arrêta en Nouvelle-Calédonie.

\section{Des années 1850 aux années 1950: un siècle d'intense travail international d'inventaire de la flore néo-calédonienne}

Il faut ensuite attendre la prise de possession officielle par la France en 1853 pour que reprennent des explorations botaniques de la NouvelleCalédonie. La seconde moitié du $\mathrm{XIX}^{\mathrm{e}}$ siècle apparaît d'ailleurs particulièrement riche de ce point de vue avec la constitution de nombreux herbiers et l'envoi de dizaines de milliers d'échantillons à l'herbier du Muséum national d'histoire naturelle de Paris. Par exemple, entre 1868 et 1872, le botaniste et explorateur Benjamin Balansa envoya près de quatre mille échantillons à l'herbier du Muséum (O'Reilly, 1955 : 60). Jean Pancher, lui aussi botaniste, envoya également entre 1861 et 1870 de très nombreuses graines, bois et plantes sèches au Muséum, qui récupéra son herbier personnel après sa mort en 1879 (O'Reilly, 1955 : 78). Ce travail d'inventaire effectué par des collecteurs, dont certains sont restés anonymes alors que d'autres ont tiré de ces travaux une certaine notoriété, s'est traduit par la constitution de nombreux herbiers, dont le plus célèbre est celui du Muséum, ainsi que par la création d'un jardin botanique à Nouméa en 1863. Herbiers et jardins botaniques permettent l'archivage des échantillons naturels, morts ou vivants, et donc leur description minutieuse, étape essentielle dans le processus de (re)connaissance de la flore. Ce travail descriptif donne lieu à une intense activité de publication scientifique. Dans son chapitre « Botanique », Patrick O'Reilly (1955) dénombre soixante-seize auteurs pour la période considérée (seconde moitié du $\mathrm{XIX}^{\mathrm{e}}$ ) dont trente-sept Français, quinze Anglais, Écossais ou Australiens, treize Allemands ou Autrichiens, neuf Belges et deux Suisses. La dimension internationale est frappante : plus de la moitié des auteurs ayant publié dans la 
seconde moitié du $\mathrm{XIX}^{\mathrm{e}}$ siècle un article sur la botanique de la Nouvelle-Calédonie ne sont pas français. La connaissance de la nature néocalédonienne et, plus particulièrement, de la flore s'est donc d'emblée élaborée sur une scène internationale au sein de revues scientifiques de langues anglaise, allemande et française.

«En 1862, trois officiers du corps de la chirurgie de Marine nous ont donné deux mémoires développés sur l'île et sa population indigène, qui sont au nombre des meilleures études que l'on ait publiées sur le monde océanien: l'un, de M. Rochas dans un volume particulier ( $\left.\mathrm{n}^{\mathrm{o}} 204\right)$; les deux autres, MM. Vieillard et Deplanche, dans un travail écrit en commun pour la Revue du ministère de la Marine ( $\mathrm{n}^{\circ}$ 205).» (L’Année géographique, 1862 : $335 \mathrm{http}: / /$ visualiseur.bnf.fr/Visualiseur?Destination= Gallica\&O=NUMM-104728)

C'est sur la partie botanique de l'essai de Vieillard et Deplanche que je voudrais m'arrêter maintenant.Ce volet botanique s'inscrit dans le cadre plus global des « Essais sur la NouvelleCalédonie » écrits par ces deux auteurs et qui s'intéressent aux différentes facettes de la vie sociale, économique, politique et culturelle des « indigènes » (histoire, géographie, topographie, sol, population, caractère, religion, costumes, anthropophagie, organisation politique, maladies, conditions des femmes, propriété de la terre, chasse, pêche, alimentation, zoologie, etc.). Une édition récente de cet essai a été réalisée dans la collection Fac-similés océaniens de Frédéric Angleviel en 2001. La partie consacrée à la botanique comporte quarante et une pages. C'est une sorte d'inventaire descriptif de différentes familles $^{6}$ et espèces végétales qui semblaient importantes aux yeux de ces deux auteurs, d'un point de vue alimentaire et/ou médicinal. La lecture en est fastidieuse, mais quelques éléments méritent d'être soulignés. Voici un exemple de la description d'une espèce d'igname, Dioscorea bulbifera, qui appartient au genre Dioscorea et à la famille des Dioscorées:

« Dioscorea bulbifera, Forst., Desmouan des indigènes. - Rhizome tubéreux de la grosseur du poing, allongé, tronqué à son extrémité inférieure, et couvert de fibrilles radiculaires ; tige grêle, cylindrique, tordue, striée, volubile à gauche ; feuilles alternes, larges, cordiformes, étalées, entières, luisantes en dessus, nervées, un peu ondulées sur les bords, et terminées en pointe scarieuse; nervures de 11 à 13. Fleurs en longs épis axillaires ou terminaux, réunis deux à quatre ensemble ; périgone petit, violacé ; capsule dressée, rigone, comprimée ; loges à deux graines ailées. L'aisselle des feuilles supérieures donne presque toujours naissance à des turions plus ou moins volumineux, souvent de la grosseur d'un œuf; ces tubercules sont grisâtres, rugueux, bosselés, et présentent des yeux comme la Pomme de terre. Lorsque les bonnes espèces d'ignames commencent à manquer, les femmes recueillent les tubercules et les turions de cette plante, et les mangent, après les avoir soumis au lavage pour leur enlever le principe âcre qu'ils contiennent; à cet effet, elles les râpent grossièrement, et elles en emplissent les petites corbeilles dont nous avons parlé, qu'elles suspendent pendant quelques heures au-dessous d'un filet d'eau. Le Dioscorea bulbifera est très commun et très recherché à une certaine époque de l'année. »

On distingue clairement deux parties dans cette description. La première est une diagnose dans laquelle les deux auteurs se livrent à une description extrêmement minutieuse de la plante concernée. La deuxième partie contient la description approchée de certains usages alimentaires qui en sont faits. La lecture de la diagnose par un lecteur non botaniste procure la sensation étrange de n'y rien comprendre, comme à la lecture d'une langue étrangère dont se dégage néanmoins une poésie littéraire qui n'est pas sans évoquer certains passages de Georges Pérec. Aux yeux d'une ethnographe, l'aspect le plus remarquable est l'extrême précision de la description de la plante, une sorte d'orfèvrerie descriptive. L'objectif de cette description est la reconnaissance: les botanistes s'attachent à décrire minutieusement chacune des caractéristiques de la plante pour la distinguer des autres et pouvoir ainsi l'identifier avec certitude. La somme de ces descriptions constitue la carte d'identité de la plante. Tout naturaliste qui en verra un autre spécimen sera capable de reconnaître qu'il appartient à la même espèce car il présente les mêmes caractéristiques.

La manière de libeller le nom d'une plante mérite aussi d'être souligné. Aux côtés des noms de genre et d'espèce rédigés en latin figure le nom ou une abréviation du nom du naturaliste qui l'a, le premier, découverte et nommée, en l'occurrence Forst. pour Forster. Cette manière de nommer se situe dans la tradition du système de classification binomial de Linné.

« C'est pour faciliter la mémorisation et la désignation sur le terrain que Linné introduit progressivement ces "binômes" encore en usage aujourd'hui et qui, à chaque espèce, attribuent un nom générique [un nom de genre] et un adjectif ou un substantif spécifique [c'est-à-dire un nom d'espèce]. » (Drouin, 1997 : 489)

6. Chaque être vivant appartient à une espèce qui appartient elle-même à un genre qui appartient lui-même à une famille. La famille est donc l'unité taxonomique qui se situe au-dessus de celles du genre et de l'espèce. Par exemple : l'homme appartient à l'espèce sapiens qui appartient au genre homo qui appartient à la famille des Hominidés. 
De très nombreuses espèces illustrées par Vieillard et Deplanche ont déjà un nom de découvreur ou d'inventeur ce qui montre bien qu'en 1862 la végétation néo-calédonienne était déjà en partie connue, nommée, décrite et classée par les naturalistes européens. Les noms d'inventeurs qui reviennent le plus souvent sont : Forster, Labillardière, Gaudin, pour ne parler que des naturalistes qui ont exploré directement la Nouvelle-Calédonie. Lorsqu'un naturaliste observe le premier en Nouvelle-Calédonie une espèce de plante déjà connue ailleurs, donc déjà nommée et décrite, la plante ne portera pas son nom. Seul reste le nom de celui qui l'a nommée, décrite et reconnue le premier où que ce soit dans le monde. Dans la seconde moitié du XIX ${ }^{\mathrm{e}}$ siècle, la flore néo-calédonienne a ainsi beaucoup été mise en mots (diagnose).

L'arrivée, en 1868, de Benjamin Balansa sur le territoire néo-calédonien constitue un tournant dans l'histoire de la botanique. Selon Hugh Mac Kee, l'un des plus grands collecteurs de la flore néo-calédonienne dans la seconde moitié $\mathrm{du} \mathrm{Xx}^{\mathrm{e}}$ siècle :

« jusqu'à cette époque, on a l'impression d'une activité botanique débordante mais assez décousue. Des collecteurs nombreux prospectèrent les régions de l'archipel qui leur furent accessibles; quelques-uns étudièrent leurs récoltes assez soigneusement pour donner aux nouvelles espèces des noms valables basés sur des descriptions techniques suffisantes. Après 1868 , les choses furent beaucoup mieux organisées ; le Muséum national d'histoire naturelle à Paris envoya le botaniste voyageur Balansa qui séjourna en NouvelleCalédonie presque cinq ans (1868-1872). Les taxonomistes du Muséum (Baillon, Brongniart et Gris) classèrent ses récoltes, qui révélèrent progressivement toute la grande originalité de la flore canaque, et en décrivirent les nouveautés. La collection des plantes calédoniennes du Muséum est de loin la meilleure du monde et les échantillons de Balansa en restent le cadre central. » (Mac Kee, 1964 : 23)

\section{Hugh Mac Kee précise que :}

« son monument restera ses spécimens plutôt que ses écrits, quoique son œuvre systématique sur les Graminées, sa famille de prédilection, conserve une valeur permanente. » (1964:24)

\section{Et il souligne qu'après son départ :}

« l'exploration botanique de la Nouvelle-Calédonie continua au ralenti jusqu'à la fin du $\mathrm{XIX}^{\mathrm{e}}$ siècle. » (1964 : 24)

La première moitié $\mathrm{du} \mathrm{xx}^{\mathrm{e}}$ siècle se caractérise par une présence soutenue de botanistes étrangers, de langue allemande, anglaise et italienne, aux côtés d'explorateurs français. Patrick
O'Reilly en dénombre quatre-vingt-onze dont trente-deux Français ou Belges, vingt-huit Allemands, Autrichiens ou Suisses allemands, vingtsix Anglais, Australiens, Néo-Zélandais, Américains, Sud-Africains ou Japonais et cinq Italiens. Quelques grands botanistes ont joué un rôle majeur dans les progrès de la botanique de cette période.

Schechter, grand botaniste voyageur allemand, fit en Nouvelle-Calédonie en 1902-1903 une récolte considérable dont il publia, aidé de quelques systématiciens de l'Herbier de Berlin, une énumération très importante comprenant la description de plantes jusqu'alors inconnues (Mac Kee, 1964 : 24). Les récoltes faites par Fritz Sarasin et Jean Roux en 1911 et 1912 ont conduit à la publication, entre 1914 et 1921, d'un ouvrage collectif en plusieurs volumes intitulé Nova Caledonia ; des botanistes de langue allemande, italienne et française ont écrit dans cet ouvrage. A. U. Däniker, professeur à l'université de Zürich, a beaucoup publié en allemand, dans les années 1920-1930 sur la végétation néocalédonienne.

Hugh Mac Kee (1964 : 25) remarque que cette exploration botanique s'est accélérée après 1945 avec des botanistes d'origines très variées (Australie, États-Unis, Nouvelle-Zélande, plusieurs pays européens) qui sont venus nombreux pour étudier la flore kanak et en récolter des échantillons. Mais il souligne qu'il s'agissait souvent de spécialistes venus pour chercher pendant de courtes visites des plantes d'une seule famille, parfois d'un seul genre. La seule expédition importante des années récentes, poursuit Mac Kee (1964: 25), est la Mission franco-suisse (Guillaumin, Baumann-Bodenheim et Hürlimann) qui, en 1950-1952, parcourut l'archipel en tous sens, réunissant une collection formidable de plantes de toutes sortes et de toutes les régions. Et, ajoute-t-il, de gros fascicules des résultats de cette mission sont parus régulièrement sous la plume de Guillaumin. Botaniste français, professeur au Muséum national d'histoire naturelle, André Guillaumin a beaucoup contribué à la connaissance de la flore de la Nouvelle-Calédonie et à sa révision.

L'étude scientifique de la flore néo-calédonienne a donc été très active et productive entre le milieu du XIX ${ }^{\mathrm{e}}$ siècle et le milieu du $\mathrm{Xx}^{\mathrm{e}}$. Dans les années 1950, un savoir naturaliste international existait déjà sur la biodiversité végétale de la Nouvelle-Calédonie. Comme le souligne un écologue américain contemporain, Peter Lowry, la flore néo-calédonienne est bien connue et documentée depuis longtemps, en comparaison des autres régions tropicales. Je le cite : 
« Depuis le travail pionnier des Forster qui accompagnèrent le Capitaine Cook lors de la première expédition européenne qui visita la Nouvelle-Calédonie, celle-ci a reçu beaucoup d'attention de la part des collecteurs de plantes, et ce jusqu'à aujourd'hui. L'origine, l'évolution et l'histoire biogéographique des plantes et de la végétation endémiques de la NouvelleCalédonie ont été l'objet de très nombreuses publications au cours de ces 125 dernières années. [...] Mais la part la plus importante de l'intense exploration botanique de la Nouvelle-Calédonie s'est déroulée à partir des années 1950. » (Lowry, $1996: 2$, traduit par moi)

Jusqu'au milieu du $\mathrm{xx}^{\mathrm{e}}$ siècle, il semblerait qu'on ne trouve trace, dans les publications botaniques sur la Nouvelle-Calédonie ni d'opérations de comptage systématiques ni de données statistiques, ni de cris d'alarme. Comme au $\mathrm{XIX}^{\mathrm{e}}$ siècle, la flore est essentiellement mise en mots, sous forme de diagnoses de plus en plus raffinées et précises. Il faut attendre la seconde moitié $\mathrm{du} \mathrm{Xx}^{\mathrm{e}}$ siècle, et plus précisément les années 1970-1980, pour que s'opère un renouvellement des méthodes d'approche du couvert végétal. Ce renouvellement se caractérise par l'apparition et le développement d'approches écologiques et synthétiques qui consistent notamment à utiliser des méthodes quantitatives et des traitements statistiques permettant de caractériser les différentes formations végétales de la Nouvelle-Calédonie. Outre de nouvelles formes de connaissances sur la flore néocalédonienne, cette approche quantitative de la nature et ces traitements statistiques vont permettre, aux côtés du développement de discours alarmistes et menaçants fondés sur des données mesurables et donc indiscutables, l'élaboration et la mise en œuvre d'actions politiques de conservation de la nature. La mise en politique de ces nouvelles formes de savoirs scientifiques va s'opérer principalement dans les années 19902000.

\section{De la mesure de la biodiversité à sa conservation : la seconde moitié du $\mathrm{xx}^{\mathrm{e}}$ siècle}

En 1946, l'Institut français d'Océanie est créé à Nouméa. Il deviendra l'ORSTOM en 1964 (Office de recherche scientifique et technique outremer), puis l'IRD (Institut de recherche sur le développement) en 1998. Dans la seconde moitié du $\mathrm{Xx}^{\mathrm{e}}$ siècle, c'est au sein de cette institution scientifique que sera conduite la majorité des recherches sur la nature néo-calédonienne. Avec l'implantation de l'IFo, débutent des inventaires écologiques de la flore et des plantes médicinales. Ceux-ci vont, par exemple, conduire à la création de l'herbier de Nouvelle-Calédonie en 1963 (Lowry, 1996).

Les naturalistes anglo-saxons, qui avaient publié quelques rares articles dans la première moitié $\mathrm{du} \mathrm{xx}^{\mathrm{e}}$ siècle, investissent plus massivement le terrain néo-calédonien grâce, notamment, à l'énorme travail de collecte réalisé par Hugh Mac Kee, considéré comme le plus grand collecteur de plantes de la Nouvelle-Calédonie, ainsi qu'au rôle joué par plusieurs écologues du Missouri Botanical Garden. Selon Peter Lowry, entre les années 1960 et sa mort en 1995, Hugh Mac Kee a collecté environ quarante-cinq mille échantillons. En 1996, Peter Lowry estime qu'il y a entre deux cent mille et deux cent cinquante mille spécimens collectés en NouvelleCalédonie. Ce matériel sera utilisé pour la célèbre Flore ${ }^{7}$ de la Nouvelle-Calédonie et Dépendances, publiée par le Muséum national d'histoire naturelle. En 1996, la Flore couvrait quarante-cinq familles et mille deux cent quatrevingt-une espèces soit presque $27 \%$ des quelque quatre mille sept cent quatre-vingts espèces de plantes vasculaires présentes sur le territoire dont mille quatre cents ont été introduites ou cultivées et dont trois mille trois cent quatrevingts sont considérées comme endémiques. En tout, presque $70 \%$ de la flore néo-calédonienne serait classée par les opérations taxonomiques modernes (Lowry, 1996).

Dans les années 1970 et 1980, la majorité des recherches sur la flore en Nouvelle-Calédonie sont conduites au sein de l'orstom par JeanMarie Veillon, Tanguy Jaffré et Philippe Morat. Ces trois botanistes et écologues ont joué un rôle majeur dans la connaissance des différentes formations végétales de la Nouvelle-Calédonie et, notamment, dans la connaissance des forêts sclérophylles $^{8}$ (ou forêts sèches). Dans les années 1990 , ce sont ces forêts qui seront mises sur le devant de la scène internationale de la conservation des groupements végétaux menacés et constituées en hotspot de la biodiversité forestière tropicale mondiale. C'est la chronique de cette histoire que je vais maintenant retracer.

\section{De 1956 à 1981 : l'invisibilité sociale et politique des forêts sèches}

Avant 1981, ces forêts étaient socialement et politiquement invisibles. Elles n'avaient

7. Les Flores permettent de rassembler en un ou quelques volumes les travaux dispersés des botanistes et constituent un outil indispensable pour qui veut (re)connaître la végétation d'une aire biogéographique donnée.

8. Scleros veut dire « dur » en grec. Les arbres de ces forêts ont des feuilles assez dures. 
d'ailleurs même pas de nom. Elles n'étaient pas distinguées d'autres types de formations forestières. C'étaient des forêts. C'est l'attribution d'un nom à cet objet naturel qui va commencer à le distinguer des autres formations forestières et ainsi le faire exister socialement en lui donnant une singularité, une unicité et une identité. C'est en 1981 que les forêts sèches sont pour la première fois distinguées et appelées alors du terme de "forêts sclérophylles » dans l'atlas que les botanistes de l'ORSTOM sont alors en train de rédiger. Deux facteurs permettent de comprendre pourquoi, en 1981, ces forêts ont été identifiées et ainsi dénommées.

D'abord, un travail précurseur avait été fait en 1956 par un naturaliste, Robert Virot, qui fut le premier, en Nouvelle-Calédonie, à ne pas faire uniquement de la taxonomie et de la systématique. À cette époque-là, Robert Virot réalise un travail pionnier de phytosociologie et de caractérisation de certaines formations végétales. Et c'est lui qui, le premier, considère que les forêts situées en bord de mer, à basse altitude, sur la côte ouest de la Grande Terre, forment une unité de végétation qu'il appelle alors « bois ou forêts littorales ». Mais son travail reste d'abord sans suite. Il était cependant bien connu des botanistes de l'ORSTOM. Et, lorsque en 1981, ils constituent l'atlas de la végétation de NouvelleCalédonie, ils reprennent les résultats de Virot en les complétant par la connaissance que l'un d'entre eux, Philippe Morat, avait des forêts sclérophylles de Madagascar où il venait de passer douze années. Ce botaniste et systématicien qui venait d'arriver en Nouvelle-Calédonie et de prendre la direction du laboratoire de Botanique de l'oRsTOM avait observé des similitudes entre les « forêts littorales » décrites par Robert Virot et les forêts sclérophylles de Madagascar. Il décida d'appeler ces "forêts littorales" de Nouvelle-Calédonie, «forêts sclérophylles ». En 1981, ces forêts sont donc nommées et cartographiées. Elles sont ainsi distinguées du reste de la nature néo-calédonienne et dotées d'une existence et d'une identité propres.

\section{Les années 1980 : un tournant dans la botanique en Nouvelle-Calédonie}

Philippe Morat ne se contenta pas de qualifier ces forêts. Il contribua, avec d'autres, à donner une nouvelle impulsion aux recherches botaniques menées en Nouvelle-Calédonie. Jusqu'à la fin des années 1970, mis à part le travail pionnier de Robert Virot, ces recherches avaient uniquement consisté en des travaux de taxonomie et de systématique. L'extrême richesse et l'originalité de la flore néo-calédonienne, liées notamment à la nature de ses sols $(30 \%$ des sols de la Nouvelle-Calédonie sont des sols miniers) avaient longtemps conduit les botanistes à identifier, décrire et classer les espèces végétales qu'ils collectaient et à les conserver dans les principaux herbiers du monde et notamment dans celui du Muséum national d'histoire naturelle à Paris. L'entreprise taxonomique et systématique constituait un travail de très longue haleine en raison précisément de cette richesse floristique et de son niveau très élevé d'endémisme. Mais à la fin des années 1970 et, plus encore, dans les années 1980, la direction de l'ORSTOM demanda à ses botanistes de diversifier leurs approches et de conduire des recherches plus synthétiques et plus écologiques. Elle leur demanda également de s'intéresser davantage à des questions de développement et aux milieux naturels anthropisés.

Dans les années 1980, ces chercheurs se mirent alors à caractériser les différentes formations végétales de la Nouvelle-Calédonie, en commençant par les forêts humides, puis les maquis miniers et enfin les forêts sèches à la fin des années 1980. Ces forêts sont situées en bord de mer et incluses dans de grandes propriétés d'élevage extensif. En raison de cette localisation dans des milieux anthropisés, les botanistes pensaient que ces forêts n'étaient pas intéressantes. Ils considéraient que ces milieux ne pouvaient plus receler d'espèces végétales dignes d'intérêt et que, s'il y en avait eu, les précédents botanistes les avaient nécessairement découvertes, étant données l'ancienneté et la densité de l'exploration botanique de la Nouvelle-Calédonie.

Or, au cours d'une exploration dans une forêt sèche située sur la réserve spéciale de chasse du haut-commissaire sur l'îlot Leprédour, ils découvrirent en 1988, trois pieds seulement d'une espèce unique au monde qu'ils appelèrent Pittosporum tanianum Veillon et Tirel (du nom des deux découvreurs). Cette découverte inattendue provoqua un vif émoi dans la communauté des botanistes. En effet, cette réserve spéciale de chasse du haut-commissaire était une zone explorée depuis longtemps par les botanistes et une zone anciennement "perturbée » par les activités humaines. De plus, les chercheurs ne découvrirent que trois pieds subsistants de cette espèce inconnue. Ils eurent alors l'intime conviction, d'une part, que cette espèce était en voie d'extinction et, d'autre part, qu'elle était un indice de la richesse floristique potentielle de ces forêts sèches qu'ils avaient jusqu'alors négligées. Les botanistes de l'ORSTOM réussirent à convaincre la province Sud, créée par les accords de Matignon (1988) et dotée de toute compétence 
en matière d'environnement et de développement pour le Sud de la Nouvelle-Calédonie, de l'importance de cette découverte. Elle leur commandita et finança les premiers inventaires floristiques dans les forêts du Sud de la NouvelleCalédonie. 1988 est une date doublement importante pour la mise en politique des savoirs scientifiques naturalistes sur les forêts sèches puisque, outre la découverte de Pittosporum tanianum, c'est la date de parution de l'article de Norman Myers qui faisait de ces forêts de NouvelleCalédonie un des dix hotspots de la biodiversité mondiale. La notion de hotspot devait servir à identifier les zones prioritaires pour les politiques de conservation à l'échelle mondiale.

Les années 1990 : premiers inventaires floristiques, alerte internationale et premiers accords de conservation

Les premiers inventaires floristiques des forêts sèches de la province Sud furent donc réalisés en 1990-1991. Ils donnèrent lieu à la rédaction d'un rapport (Jaffré et Veillon, 1991) et de plusieurs articles scientifiques (Jaffré, Morat et Veillon, 1993). L'événement majeur des années 1990 fut le constat, en 1993, de la disparition des trois pieds de Pittosporum tanianum et le récit, en 1995, de ce constat dans un article scientifique paru dans une prestigieuse revue anglo-saxonne de biologie de la conservation, Biodiversity and Conservation.

\section{- L'alerte internationale}

Les botanistes considèrent alors que Pittosporum tanianum est une espèce disparue, la probabilité de la trouver dans d'autres forêts sèches de Nouvelle-Calédonie étant infime. Ils prospectent le territoire néo-calédonien depuis bien longtemps et ils auraient trouvé d'autres spécimens de cette espèce s'il en existait. Or, le fait qu'ils n'aient découvert que trois exemplaires (et) aussi tardivement qu'en 1988 montre déjà l'extrême rareté de sa présence. Ainsi, Pittosporum tanianum a le triste privilège, pensent-ils alors, de constituer la première disparition d'espèce néocalédonienne connue et documentée. Le constat de cette disparition va jouer un rôle décisif et constituer un tournant. Elle va en effet conduire les botanistes de l'ORSTOM à publier en 1995 un article en anglais, dans un " style conservation ", dans la revue Biodiversity and Conservation. C'est un malacologue ${ }^{9}$ du Muséum national d'histoire naturelle de Paris, Philippe Bouchet, qui en a pris l'initiative. Il connaissait bien les forêts néo-calédoniennes et il était personnellement engagé dans des instances internationales de la conservation (au sein de la Commission de sauvegarde des espèces menacées de l'UICN). En co-signant cet article avec ses collègues botanistes de l'ORSTOM, il a voulu les aider à donner une audience internationale à leurs travaux sur les forêts sèches qui restaient jusqu'alors uniquement connus en Nouvelle-Calédonie. Dans cet article, ils dévoilent la disparition de Pittosporum tanianum, dont ils joignent la description en annexe. Et ils insistent sur les risques majeurs de disparition des dernières forêts sèches de Nouvelle-Calédonie. Dans un entretien, Philippe Bouchet explique que le "style conservation » consiste à sortir du schéma « les botanistes parlent aux botanistes de l'intérêt scientifique d'un taxon " pour, d'une part, s'adresser à un autre public non spécialiste du dit taxon et, d'autre part, adopter un ton qui insiste avant tout sur la menace et les dangers de disparition de ce taxon. Cet article a eu l'effet recherché puisque, quelques mois seulement après sa parution, un des directeurs scientifiques du WWF États-Unis, Eric Dinerstein, contacta personnellement Philippe Bouchet pour qu'il lui expose le cas des forêts sèches de Nouvelle-Calédonie. Eric Dinerstein fut dès lors convaincu de l'urgence d'agir pour conserver ces forêts. Dans ce but, il mobilisa le WwF - France à qui il demanda de prendre en charge cette question.

$\mathrm{Si}$ la question des forêts sèches néocalédoniennes a trouvé un écho immédiat auprès du WwF, c'est aussi parce que, à cette même époque, dans la seconde moitié des années 1990, le WWF s'était engagé dans une nouvelle stratégie à l'échelle mondiale, consistant à identifier les principales éco-régions de la planète. En se basant sur les travaux existants de biogéographie qui avaient cartographié les principaux biomes et écozones (régions biogéographiques) de la planète, le WWF cherchait alors à produire une nouvelle cartographie de la végétation mondiale basée sur une nouvelle délimitation : les écorégions. Ils en identifièrent 238. Ces 238 écorégions constituent les exemples les plus remarquables et les plus représentatifs de la diversité des écosystèmes mondiaux, ainsi que des régions prioritaires pour des actions de conservation. Parmi ces 238 éco-régions du monde, quatre se trouvent en Nouvelle-Calédonie, dont l'écorégion « forêts sèches"

En 1997, des représentants du WWF - France viennent en Nouvelle-Calédonie pour exposer cette nouvelle stratégie de conservation basée 
sur la notion d'éco-régions et pour évoquer plus particulièrement la question de la conservation des forêts sèches. Jusqu'en 2001, date de la création du Programme de conservation des forêts sèches de Nouvelle-Calédonie, le WwF - France va organiser plusieurs missions et rédiger plusieurs rapports qui vont servir à préparer et à structurer ce programme de conservation.

\section{- Les premières mesures conservatoires}

Parallèlement à ce processus d'alerte internationale, une découverte effectuée en 1994 par les botanistes de l'ORSTOM dans une forêt sèche de la province Sud de Nouvelle-Calédonie va déboucher sur le premier accord de conservation entre la province Sud et le propriétaire privé de cette forêt, Claude Metzdorf. En effectuant des relevés dans cette forêt, les botanistes de l'ORSTOM découvrent une Euphorbiacaea d'un genre inconnu en Nouvelle-Calédonie, Trigonostemon. Ils eurent la chance, raconte dans un entretien l'un de ces botanistes, Jean-Marie Veillon, que ce genre ait été tout juste l'objet d'une révision et d'une mise à jour par un chercheur asiatique. Ils purent ainsi rapidement s'apercevoir que l'espèce trouvée dans la forêt de Claude Metzdorf était inconnue et qu'elle était une espèce endémique. Outre la découverte de cette nouvelle espèce, ils découvrirent, dans cette même forêt, de nombreuses espèces végétales à distribution limitée et rassemblées dans un mouchoir de poche. Ils en déduisirent que cette forêt était une relique d'une ancienne forêt sèche importante. Étant donné que cette relique était située au cœur de l'exploitation d'élevage de Claude Metzdorf, Jean-Marie Veillon voulut immédiatement protéger cette forêt du bétail et des cerfs et il parvint aisément à convaincre Claude Metzdorf, qu'il connaissait bien, et la province Sud de l'urgence de clôturer ces huit hectares de forêts. La mise en défens est une mesure de conservation très souvent utilisée, dans les cas d'urgence notamment.

«Le 12 juillet 1994, les autorités de la province Sud concluent une convention avec Mr. Metzdorf, éleveur dans la commune de Poya sud : la convention vise la protection de huit hectares de forêt sclérophylle, situés sur sa propriété. L'accord octroie à l'éleveur une somme de 1200000 FCFP [soit $10000 €$ ] pour l'édification d'une clôture en vue de la protection de la forêt sclérophylle ; l'accord impose aussi des spécifications techniques complètes sur la hauteur de la clôture, la densité du maillage ou concernant la nécessité qu'un fil de fer soit placés au ras du sol. Le but d'édification de la clôture, souligné par l'accord, est d'empêcher la circulation des animaux domestiques ou sauvages dans la zone protégée. L'éleveur s'engage quant à lui à réaliser les travaux dans une période de douze mois suivant la conclusion de l'accord. On observe que la somme correspond au paiement pour service rendu par l'éleveur, plutôt qu'à une compensation pour non utilisation du terrain protégé. » (Giraud-Kinley, 1998 : 388)

Pourquoi Claude Metzdorf a-t-il accepté de perdre l'usage d'une partie de sa propriété ?Quel était son intérêt? Et comment Jean-Marie Veillon l'a-t-il convaincu de mettre sa forêt en défens pour une période de dix ans?

Les forêts sèches ne sont pas exploitées par les éleveurs. Elles constituent simplement pour le bétail en période sèche des abris dans lesquels il trouve des compléments alimentaires. Mais elles ne sont pas au cœur de leur système d'élevage. De plus, d'après Jean-Marie Veillon, le simple fait d'apprendre à Claude Metzdorf qu'il possédait dans ses forêts des espèces végétales uniques au monde, l'a littéralement enthousiasmé. Sa forêt a dès lors pris une " valeur morale ", raconte JeanMarie Veillon dans un entretien. Ce modèle d'accord de conservation signé entre un propriétaire privé et les gestionnaires des forêts sèches sera repris par le programme de conservation des forêts sèches à partir de 2001, dont il constitue d'ailleurs le seul dispositif de conservation.

\section{En guise de conclusion}

Les textes scientifiques qui, dans les années 1990, ont eu comme sujet les forêts sèches néocalédoniennes, associent presque toujours des données ou des sources d'origines diverses: inventaires et comptage d'espèces, traitements statistiques, propos alarmistes, insistance sur le caractère remarquable de la biodiversité néocalédonienne, références à des classements internationaux (hotspots et éco-régions). Ces articles s'ouvrent généralement sur des données chiffrées et des pourcentages qui servent à montrer d'emblée le caractère remarquable de la biodiversité néo-calédonienne et les menaces qui pèsent sur les forêts sèches :

« La Nouvelle-Calédonie a été désignée comme un hotspot de la biodiversité avec 3261 espèces de plantes indigènes dont 74,3\% sont endémiques. [...] Les forêts tropicales sèches sont les plus menacées de tous les types de végétation terrestre de la Nouvelle-Calédonie parce qu'elles occupent moins de $2 \%$ de leur surface d'origine à cause des défrichements, de l'élevage et des feux. » (Gillespie et Jaffré, 2002 : 1687-1697, traduit de l'anglais par moi) 
La précision de ces chiffres et de ces pourcentages est censée fonder le caractère scientifique de leur acquisition et donc leur véracité et leur légitimité. Trois mille deux cent soixante et une espèces de plantes indigènes ont été dénombrées en Nouvelle-Calédonie. Les différents niveaux de menace qui pèsent sur ces différentes espèces, selon les classifications établies par l'UiCN, sont souvent indiqués. Si la précision de ces comptages, de ces opérations statistiques et de ces chiffres sert de toute évidence les propos alarmistes de certains articles, c'est aussi parce que ces alertes sont étayées par des données vérifiables scientifiquement. Elles possèdent ainsi une réelle efficacité pour atteindre leurs objectifs de mobilisation des pouvoirs publics et des bailleurs de fonds afin qu'ils mettent en œuvre des actions de conservation.

Pourtant, de nombreux écologues soulignent le caractère critiquable de la notion même de biodiversité et du caractère quantitatif de sa mesure, notamment des inventaires floristiques qui sont à la base de cette mesure. Odile Poncy et Jean-Noël Labat soulignent par exemple le fait que «l'insuffisance des données d'inventaire peut conduire à des artefacts lors de l'évaluation de la richesse spécifique, ou des taux d'endémisme dans des régions données » (1996:13). Ils constatent que les prospections botaniques ont été souvent organisées et financées à la faveur de programme de destruction des milieux naturels concernés (projets routiers, industriels, etc.). Les lacunes et les ignorances sur une masse considérable de la flore tropicale et les incertitudes qui en découlent, soulignées notamment par Odile Poncy et Jean-Noël Labat, ne sembleraient donc pas constituer des obstacles à la mise en place de politiques de conservation de la nature et de la biodiversité. L'action politique et son financement, la gestion de la nature et de sa biodiversité, ne s'ancrent pas toujours dans un savoir scientifique bien stabilisé.

\section{Remerciements}

Je remercie chaleureusement Christian Papineau, le directeur du programme de Conservation des forêts sèches, de m'avoir apporté une aide précieuse dans la préparation et le déroulement des enquêtes que j'ai effectuées en octobre 2005 en Nouvelle-Calédonie. Je remercie également Philippe Bouchet, Tanguy Jaffré, Philippe Morat, Bernard Suprin et Jean-Marie Veillon pour l'aide qu'ils m'ont apportée pour la compréhension de l'histoire de ce programme de conservation ainsi que de celle de la botanique en Nouvelle-Calédonie. Et je remercie enfin tous les participants du programme forêts sèches qui ont tous accepté de répondre à mes questions.

\section{BIBLIOGRAPHIE}

Blanckaert Claude, Claudine Cohen, Pietro Corsi et Jean-Louis Fischer (éds.), 1997. Le Muséum au premier siècle de son histoire, Paris, éditions du MNHN, $687 \mathrm{p}$.

Bouchet Philippe, Tanguy JAFfré et Jean-Marie VeILlon, 1995. Plant extinction in New Caledonia: protection of sclerophyll forests urgently needed, Biodiversity and Conservation 4, pp. 415-428.

Bourguet Marie-Noëlle, 1997. L'histoire naturelle du $\mathrm{XVII}^{\mathrm{e}}$ au XIX ${ }^{\mathrm{e}}$ siècle, in C. Blanckaert, C. Cohen, P. Corsi et J.-L. Fischer (éds), Le Muséum au premier siècle de son histoire, Paris, éditions du MNHN, pp. 163-196.

Callon Michel et John Law, 1997. L'irruption des non-humains dans les sciences humaines : quelques leçons tirées de la sociologie des sciences et des techniques, in B. Reynaud (dir.), Les limites de la rationalité. Les figures du collectif, actes du colloque de Cerisy, Paris, La Découverte.

Chazeau Jean, Christophe Chevillon, Claire GarriGUE, Tanguy JAFFrÉ et al., 1994. Biodiversité et conservation en Nouvelle-Calédonie, rapport de synthèse ORSTOM, Sciences de la vie 1, $32 \mathrm{p}$.

Drouin Jean-Marc, 1997. De Linné à Darwin : les voyageurs naturalistes, in M. Serres (dir.), Éléments d'histoire des sciences, Paris, Larousse, pp. 479-501.

GillesPIE Thomas Williams et Tanguy JAFFrÉ, 2002. Tropical dry forests in New Caledonia, Biodiversity and Conservation 12, pp. 1687-1697.

GIRAUD-KINLEY Catherine, 1998. La protection de la diversité biologique dans la région du Pacifique sud : réflexions sur l'efficacité du droit international, thèse de doctorat de droit, université Paris I-Panthéon-Sorbonne, sous la direction de Laurent Lucchini.

IRD, http://www.bondy.ird.fr/pleins_textes/index-tous. htm.

JAFFRÉ Thierry et Jean-Marie VEILLON, 1991. La forêt sclérophylle de la province Sud de la NouvelleCalédonie, Nouméa, orstom-province Sud, Sci. Vie, Bota. Convention 6, 93 p.

JAFFrÉ Thierry, Philippe Morat et Jean-Marie VeILlON, 1993. Étude floristique et phytogéographique de la forêt sclérophylle de NouvelleCalédonie, Bulletin du Muséum national d'histoire naturelle $15,4^{\mathrm{e}}$ série, B, Adansonia, pp. 107-147.

LATOUR Bruno, 1997. L'irruption des non-humains dans les sciences humaines : quelques leçons tirées de la sociologie des sciences et des techniques, in $\mathrm{B}$. 
Reynaud (dir.), Les limites de la rationalité. Les figures $d u$ collectif, actes du colloque de Cerisy, Paris, La Découverte.

L'Année Géographique, 1862.

LefEuvre Jean-Claude, 2000. Programme de conservation. Forêts tropicales sèches en Nouvelle-Calédonie, document de programme, WWF, $30 \mathrm{p}$.

La forêt sèche de Nouvelle-Calédonie. Conservation et gestion durable, 2003. www.foretseche.nc.

Lowry Peter, 1996. Diversity, Endemism, and extinction in the flora of New Caledonia, http://www. mobot.org/MOBOT/research/newcaledonia/intro. html.

Mac KeE Hugh, 1964. Les étapes de la connaissance botanique de la Nouvelle-Calédonie, Phytochimie et plantes médicinales des terres du Pacifique, actes du colloque CNRS, Nouméa, pp. 19-31.

Malinowski Bronislaw, 1974. L'économie primitive des îles Trobriand, in M. Godelier (éd.), Un domaine contesté: l'anthropologie économique, Paris, Mouton.

Merle Isabelle, 1995. Expériences Coloniales. La Nouvelle-Calédonie (1853-1920), Paris, Belin.

Mittermeier Russell A., Norman Myers, Jorgen B. Thomsen, Gustavo A. B. da Fonseca, Silvio OliVIERI, 1998. Biodiversity Hotspots and Major Tropical Wilderness Areas: Approaches to Setting Conservation Priorities, Conservation Biology 12, 3, pp. 516-520.
Myers Norman, 1988. Threatened Biotas: Hot Spots in Tropical Forests, Environmentalist 8, pp. 187-208.

Olson David, Eric Dinerstein et al., 2000. The Global 200: A Representation Approach to Conserving the Earth's Distinctive Ecoregions, Conservation Science Program, World Wildlife Fund-US.

O'ReILly Patrick, 1955. Bibliographie méthodique, analytique et critique de la Nouvelle-Calédonie, Paris, Publications de la société des Océanistes 4.

PISIER Georges, 1983. La découverte de la NouvelleCalédonie par Cook, Nouméa, Publications de la Société d'études historiques de la NouvelleCalédonie 5.

Poncy Odile et Jean-Noël LABAT, 1996. État actuel de l'inventaire des flores tropicales, Le Courrier de l'Environnement 27, pp. 79-91.

Ramade François, 1993. Dictionnaire encyclopédique de l'écologie et des sciences de l'environnement, Paris, Édiscience international.

VeILlon Jean-Marie, Gilles DAGostini et Tanguy JAFFRÉ, 1999. Étude de la forêt sclérophylle de la province Nord en Nouvelle-Calédonie, Nouméa, IRD, Conventions Sciences de la vie Botanique 10, pp. 154.

Vieillard E. et E. Deplanche, 1862. Essai sur la Nouvelle-Calédonie : Botanique.

VIVIEN Franck-Dominique, 2001. Le patrimoine naturel : jeux et enjeux entre nature et société, séminaire Patrimoine, document de travail. 